\title{
CH AND OPEN SUBSPACES OF $F$-SPACES
}

\author{
ALAN DOW
}

\begin{abstract}
N. J. Fine and L. Gillman showed that, if one assumes $\mathrm{CH}$, each open subset of an $F$-space of weight $c$ is an $F$-space. In this note it is shown that this fact is equivalent to $\mathrm{CH}$.
\end{abstract}

0. Introduction. A Tychonoff space is an F-space if disjoint cozero subsets are contained in disjoint zero sets. Many interesting results about $F$-spaces of weight $c$ have been shown to be consequences of the continuum hypothesis, $\mathrm{CH}$; see for example [Pa, L, FG, W1, and W2]. Unfortunately most of these have also been shown to be equivalent to $\mathrm{CH}$. For example, in [vDvM1] it is shown that Parovicenko's characterization of $\omega^{*}$ is equivalent to $\mathrm{CH}$; in [vD1] it is shown that Woods' result, that countably compact normal $F$-spaces with only $c$ continuous real-valued functions are compact, is equivalent to $\mathrm{CH}$, and it has been shown that others are not theorems of ZFC [VDvM2 and vDvM3]. In this note it is shown that Fine and Gillman's result, each open subset of an $F$-space of weight $c$ is an $F$-space, is also equivalent to $\mathrm{CH}$. It is also true, under $\mathrm{CH}$, that each locally compact subspace with weight $c$ of an $F$-space is again an $F$-space. We show that, under $\neg \mathrm{CH}$, every infinite compact $F$-space contains a locally compact subspace of weight $c$ which is not an $F$-space.

Our notation and terminology follows that of the Gillman and Jerison text [GJ]. We list some necessary facts about $F$-spaces.

0.1. Proposition. (i) $X$ is an F-space iff each cozero subset of $X$ is $C^{*}$-embedded.

(ii) $X$ is an $F$-space iff $\beta X$ is an $F$-space.

(iii) $A C^{*}$-embedded subspace of an $F$-space is an $F$-space.

(iv) Lindelöf subspaces of $F$-spaces are $C^{*}$-embedded.

We will be using the absolute $E(X)$ of a compact space $X$. There is a canonical continuous map $k_{X}$ which maps $E(X)$, the space of maximal regular open ultrafilters, to $X$ defined by $k_{X}(u) \in \cap\left\{\mathrm{cl}_{X} U: U \in u\right\}$. For each regular open subset $U$ of $X, \overline{k^{-}(U)}$ is a clopen subset of $E(X)$ and these form a base for $E(X)$. Also if $g$ is a homeomorphism of $X$ then there is a unique homeomorphism $h$ of $E(X)$ such that $k_{X} \circ h=g \circ k_{X}$. The reader should consult the survey [W3] for these and other facts about the absolute.

Received by the editors November 23, 1981 and, in revised form, January 31, 1983.

1980 Mathematics Subject Classification. Primary 54D35.

$K e y$ words and phrases. $F$-space, $P$-space.

(C)1983 American Mathematical Society $0002-9939 / 83 \$ 1.00+\$ .25$ per page 
1. Assuming CH. In this section we shall list and prove the relevant results from [FG] for completeness. The first result does not require $\mathrm{CH}$.

1.1. TheOREM [FG]. Each union of $\omega_{1}$ cozero subsets of an F-space is again an $F$-space.

Proof. Let $X$ be an $F$-space and $U=\bigcup\left\{C_{\alpha}: \alpha<\omega_{1}\right\}$ with each $C_{\alpha}$ a cozero subset of $X$. Let $g$ be a bounded real-valued continuous function defined on a cozero subset $C$ of $U$. We shall show that $g$ extends continuously to $\hat{g}$ on $U$. Recursively define bounded real-valued continuous functions $g_{\alpha}$ on $\cup_{\beta<\alpha} C_{\beta}$ such that for $\beta<\alpha, g_{\beta} \subset g_{\alpha}$ and $g_{\alpha}$ restricted to $C \cap \cup_{\beta<\alpha} C_{\beta}$ is equal to $g$. This recursion can be carried out since, for each $\alpha<\omega_{1}, \cup_{\beta<\alpha} C_{\beta} \cup\left(C \cap C_{\alpha}\right)$ is a cozero subset of $\cup_{\beta \leqslant \alpha} C_{\beta}$ and therefore is $C^{*}$-embedded in $\cup_{\beta \leqslant \alpha} C_{\beta}$. Therefore $\hat{g}=\cup_{\alpha<\omega} g_{\alpha}$ is the desired extension of $g$.

1.2. THEOREM (CH). Each open subset of an F-space of weight $c$ is an F-space.

1.3. THEOREM (CH). Each locally compact space of weight $c$ which is a subspace of an $F$-space is itself an F-space.

Proof. Let $X$ be an $F$-space and let $Y$ be a locally compact subspace which has weight $c$. By Proposition $0.1, K=\operatorname{cl}_{\beta X} Y$ is an $F$-space. Since $Y$ is locally compact and dense in $K, Y$ is open in $K$. Clearly $Y$ can be expressed as a union of $c=\omega_{1}$ cozero subsets of $K$. By Theorem $1.1 Y$ is an $F$-space.

2. The example. In this section we shall construct an example of an $F$-space $K$ with weight $\omega_{2} \cdot c$ which has an open subspace $U$ which is not an $F$-space. We shall need the following much weakened version of a result of Negrepontis. A space $X$ is a $P$-space if each countable intersection of open subsets of $X$ is open.

2.1. Proposition. If $X$ is a P-space, then $X \times \omega$ is an F-space and $X \times \omega$ is $C^{*}$-embedded in $X \times \beta \omega$.

Proof. In fact, $X \times \omega$ is a $P$-space since it is the product of two $P$-spaces. It easily follows from the fact that $\omega$ is countable (or Lindelöf) that the projection map from $X \times \omega$ to $X$ is $z$-closed [Wa]. Therefore $X \times \omega$ is $C^{*}$-embedded in $X \times \beta \omega$ [Wa,p. 193].

Let $X$ be the set $\omega_{2}+1$ endowed with the $G_{\delta}$-topology of the ordinal space $\omega_{2}+1$. Clearly $X$ is a $P$-space and $L=\left\{\lambda \leqslant \omega_{2}: \lambda\right.$ has uncountable cofinality $\}$ is the set of nonisolated points of $X$. It is not difficult to show that $\left|C^{*}(X)\right|=\omega_{2} \cdot c$ and that $X$ is Lindelöf (see 9L in [GJ]). Let $K=\beta\left(X \times \omega^{*}\right)$.

2.2. Fact. $K$ is a compact $F$-space with weight $\omega_{2} \cdot c$.

Proof. Since $\left|C^{*}(X \times \omega)\right|=\omega_{2} \cdot c$, by Proposition $2.1\left|C^{*}(X \times \beta \omega)\right|=\omega_{2} \cdot c$. Hence the weight of $\beta(X \times \beta \omega)$ is $\omega_{2} \cdot c$. Now since $X$, and therefore, $X \times \omega^{*}$ are Lindelöf, $X \times \omega^{*}$ is $C^{*}$-embedded in $\beta(X \times \omega)$ by Proposition 0.1(iv). It follows that $K$ is an $F$-space and has weight $\omega_{2} \cdot c$.

Now let $C$ be a nonclopen cozero subset of $\omega^{*}$. Define $T=\operatorname{cl}_{K}(L \times C)$ and $U=K-T$.

2.3. Fact. $U$ is not an $F$-space. 
Proof. We shall exhibit disjoint cozero subsets $C_{0}$ and $C_{1}$ of $U$ which are not contained in disjoint zero sets of $U$. Let $E_{0}$ and $E_{1}$ be disjoint subsets of $X-L$ such that $L \subset \bar{E}_{0} \cap \bar{E}_{1}$, e.g. $E_{0}$ is the even isolated ordinals and $E_{1}$ is the odd ordinals. Let $W_{n}$, for $n \in \omega$, be clopen in $\omega^{*}$ such that $\cup W_{n}=C$. Define $V_{n}(i)=$ $\operatorname{cl}_{U}\left(E_{i} \times W_{n}\right)$ for $i=0$ and $i=1$. We show that each $V_{n}(i)$ is open. Suppose that $u \in \mathrm{cl}_{U} V_{n}(i)$ for $i=0$ or 1 .

Therefore $\mathrm{cl}_{K}\left(X \times W_{n}\right)$ is a clopen neighborhood of $u$ in $K$. Let $W$ be a clopen $K$-neighborhood of $u$ contained in $\operatorname{cl}_{K}\left(X \times W_{n}\right)$ such that $W \cap(L \times C)=\varnothing$. Since $W$ is compact there is a countable set $I$ contained in $X \backslash L$ so that $W \subset$ $\operatorname{cl}_{K}\left(I \times W_{n}\right)$. Now $\left(I \cap E_{i}\right) \times W_{n}$ is clopen in $X \times \omega^{*}$ and $\operatorname{so~}_{K}\left(\left(I \cap E_{i}\right) \times W_{n}\right)$ is a neighborhood of $u$ which is contained in $V_{n}(i)$. Therefore $V_{n}(0)$ and $V_{n}(1)$ are clopen subsets of $U$ for each $n \in \omega$. Let $C_{i}=\cup_{n} V_{n}(i)$ for $i=0,1$, these are clearly cozero subsets of $U$. We must consider two cases to show that $C_{0}$ and $C_{1}$ are not contained in disjoint zero sets of $U$.

Case $1 . \mathrm{cl}_{\omega^{*}} C$ is not the intersection of $\omega_{1}$ clopen subsets of $\omega^{*}$. Let $X_{1}=X \cap\{\alpha$ : $\left.\alpha \leqslant \omega_{1}\right\}, K_{1}=\operatorname{cl}_{K}\left(X_{1} \times \omega^{*}\right)$ and $U_{1}=K_{1} \cap U$. Since $X_{1}$ is clopen in $X, K_{1}$ is clopen in $K$ and $U_{1}$ is clopen in $U$. We can show in this case that not even $U_{1}$ is an $F$-space. Note that the weight of $K_{1}$ is $c$ but, of course, by our current assumption $c \geqslant \omega_{2}$.

Suppose that $\left(U_{1} \cap C_{i}\right) \subset Z_{i}$ for disjoint zero sets $Z_{i}, i=0,1$. It follows that for each $\alpha \in E_{i} \cap X_{1}$ there is a clopen subset, $A_{\alpha}$, of $\omega^{*}$ with $\{\alpha\} \times A_{\alpha} \subset Z_{i}$ and $C \subset A_{\alpha}$. By assumption, there is a point $y \in \cap\left\{A_{\alpha} \backslash \mathrm{cl}_{\omega^{*}} C: \alpha \in E_{0} \cup E_{1}\right\}$. Therefore $\left(\omega_{1}, y\right) \in U_{1}$, and $\left(\omega_{1}, y\right) \in Z_{0} \cap Z_{1}$ because $X_{1} \times \omega^{*}$ has the product topology. This contradicts the fact that $Z_{0}$ and $Z_{1}$ are assumed to be disjoint.

Case 2. $\mathrm{cl}_{\omega^{*}} C=\bigcap\left\{B_{\xi}: \xi<\omega_{1}\right\}$ where each $B_{\xi}$ is a clopen subset of $\omega^{*}$. We may assume that $\left\{B_{\xi}: \xi<\omega_{1}\right\}$ is closed under finite intersections and is therefore a neighborhood base for $\mathrm{cl}_{\omega^{*}} C$. Note that in this case $U_{1}$ is an $F$-space because it can be expressed as an $\omega_{1}$ union of clopen subsets of $K_{1}$.

Suppose that $C_{i} \subset Z_{i}$ for disjoint zero sets $Z_{0}$ and $Z_{1}$. Again it follows that for $\alpha \in E_{i}$ there is a clopen $A_{\alpha} \subset \omega^{*}$ such that $\{\alpha\} \times A_{\alpha} \subset Z_{i}$ and $C \subset A_{\alpha}$. For each $\alpha \in E_{0} \cup E_{1}$ choose $\xi(\alpha)<\omega_{1}$ so that $B_{\xi(\alpha)} \subset A_{\alpha}$. Therefore, for $i=0,1$; there is a set $E_{i}^{\prime} \subset E_{i}$ with cardinality $\omega_{2}$ and a $\xi_{i}<\omega_{1}$ such that $\xi(\alpha)=\xi_{i}$ for each $\alpha \in E_{i}^{\prime}$. Let $p \in\left(B_{\xi_{0}} \cap B_{\xi_{1}}\right) \backslash \bar{C}$. Then $\left(\omega_{2}, p\right) \in \bar{Z}_{1} \cap \bar{Z}_{2}$ and $\left(\omega_{2}, p\right) \in U$, which contradicts the disjointness of $Z_{0}$ and $Z_{1}$.

It is fairly well known that both Case 1 and Case 2 above are consistent with $\neg \mathrm{CH}$. This is shown, for example, in [H].

REMARK. The underlying idea behind the construction of $U$ is that $U$ is like a product of a $P$-space $X$ with an $F$-space $Y$ where $Y$ has the property that there are some cozero subsets of $Y$ with no ' $X$-limits'. However, any zero sets containing them do have ' $X$-limits'. This idea is made more precise and is employed in [D] to find a necessary condition on $F$-spaces in order that their product with any $P$-space be $F$-spaces.

3. Assuming $\neg \mathbf{C H}$. In this section we will show the opposite implications of our equivalences of $\mathrm{CH}$. We shall need a result of van Douwen's [vD2] which we shall show in a pair of lemmas. 
3.1. Lemma. The P-space $\left(\omega^{*}\right)_{\delta}$ embeds in $\beta \omega$.

Proof. Let $p \in \omega^{*}$ be arbitrary and recall the definition: $\left\{p-\lim \left(x_{n}: n \in \omega\right)\right\}=$ $\cap\left\{\overline{\left(x_{n}: n \in U\right)}: U \in p\right\}$. It is well known that $\beta\left(\omega \times \omega^{*}\right)$ can be embedded in $\omega^{*}$. For $q \in \omega^{*}$, define $f(q)=p-\lim \{(n, q): n \in \omega\} \in \beta\left(\omega \times \omega^{*}\right)$. We shall show that $Q=\left\{f(q): q \in \omega^{*}\right\}$ is homeomorphic to $\left(\omega^{*}\right)_{\delta}$ and that $f$ is a homeomorphism. Let $\left(B_{n}: n \in \omega\right)$ be clopen subsets of $\omega^{*}$. Clearly $\cup\left(\{n\} \times \cup_{k \leqslant n} B_{k}: n \in \omega\right)$ is a clopen subset of $\omega \times \omega^{*}$ so $G=\overline{\bigcup_{n}\{n\} \times \cup_{k \leqslant n} B_{k}}$ is a clopen subset of $\beta\left(\omega \times \omega^{*}\right)$. It is easy to check that $G \cap Q=f\left[\cap_{n} B_{n}\right]$; hence $f$ is open. Conversely, let $U \subset Q$ be a neighborhood of $f(q)$ in $Q$. Choose $W$, a clopen subset of $\beta\left(\omega \times \omega^{*}\right)$, so that $f(q) \in W \cap Q \subset U$. Since $f(q) \in W, W^{\prime}=\{n:(n, q) \in W\} \in p$. For $n \in W^{\prime}$, choose $B_{n}$ so that $\{n\} \times B_{n}=W \cap\left(\{n\} \times \omega^{*}\right)$. We observe that $f\left[\cap\left(B_{n}: n \in W^{\prime}\right)\right]$ $\subset W$ which shows that $f$ is continuous.

\subsection{Lemma. The space $\left(2^{c}\right)_{\delta}$ embeds in $\beta \omega$.}

Proof. Since $2^{c}$ is separable, $E\left(2^{c}\right)$ embeds in $\omega^{*}$. Therefore $\left(E\left(2^{c}\right)\right)_{\delta}$ is a subspace of $\left(\omega^{*}\right)_{\delta}$ and from the fact that $\left(\omega^{*}\right)_{\delta}$ embeds in $\omega^{*}$ it follows that $\left(E\left(2^{c}\right)\right)_{\delta}$ embeds in $\omega^{*}$. Hence it suffices to show that $\left(2^{c}\right)_{\delta}$ embeds in $\left(E\left(2^{c}\right)\right)_{\delta}$. Let $k$ be the canonical map from $E\left(2^{c}\right)$ to $2^{c}$. We shall think of $E\left(2^{c}\right)$ as the set of ultrafilters of regular open subsets of $2^{c}$. We will also let + be coordinatewise addition modulo 2 on $2^{c}$ and 0 be the identity. Choose $u_{0} \in k^{\leftarrow}(\mathbf{0})$ arbitrarily. For each $x \in 2^{c}$ we have the homeomorphism $g_{x}$ of $2^{c}$ defined by $g_{x}(y)=x+y$. There is a unique homeomorphism $h_{x}$ of $E\left(2^{c}\right)$ such that $g_{x} \circ k=k \circ h_{x}$. Let $Y=\left\{h_{x}\left(u_{0}\right): x \in 2^{c}\right\} \subset\left(E\left(2^{c}\right)\right)_{\delta}$. To show that $Y$ is homeomorphic to $\left(2^{c}\right)_{\delta}$ via $k$ it suffices to show that if $A$ is clopen in $E\left(2^{c}\right)$ then $k(A \cap Y)$ is open in $\left(2^{c}\right)_{\delta}$. To this end let $U \subset 2^{c}$ be regular open. Since $2^{c}$ is ccc (there are no uncountable cellular families of open sets), there are countably many basic clopen sets $A_{n}$ such that $\cup A_{n} \subset U$ is dense. Let $F_{n}, n \in \omega$, be finite subsets of $c$ such that $A_{n}$ is only restricted on the coordinates in $F_{n}$ and let $F=\cup_{n} F_{n}$. Suppose that $x, y \in 2^{c}$ are such that $\left.x\right|_{F}=\left.y\right|_{F}$, we shall show that $k^{\leftarrow}(x) \in \overline{k^{\leftarrow}(U)}$ iff $k^{\leftarrow}(y) \in \overline{k^{\leftarrow}(U)}$. Indeed, $k^{\leftarrow}(x) \in \overline{k^{\leftarrow}(U)}$ iff $U \in h_{x}\left(u_{0}\right)$ iff int $\overline{\bigcup_{n} A_{n}} \in h_{x}\left(u_{0}\right)$ iff int $\overline{\cup\left(A_{n}+x\right)} \in u_{0}$ iff int $\overline{U\left(A_{n}+x+y\right)} \in h_{y}\left(u_{0}\right)$ iff int $\overline{\cup A_{n}} \in h_{y}\left(u_{0}\right)$ (since $\left.\left.x\right|_{F}=\left.y\right|_{F}\right)$ iff $k^{-}(y) \in \overline{k^{-}(U)}$. Now $F$ is countable so $2^{F}$ is homeomorphic to $2^{\omega}$ which is first countable. It follows that $\left.x\right|_{F} \times 2^{c-F}$ is clopen in $\left(2^{c}\right)_{\delta}$ for each $x \in 2^{c}$. Finally,

$$
k\left(\overline{k^{\leftarrow}(U)} \cap Y\right)=\bigcup_{n} A_{n} \cup \cup\left\{\left.x\right|_{F} \times 2^{c-F}: x \in k\left(\overline{k^{\leftarrow}(U)}\right)\right\}
$$

which is open by the above.

\subsection{Corollary $(\neg \mathrm{CH})$. The space $K$ constructed in $\S 2$ embeds in $\omega^{*}$.}

Proof. Recall that $X=\left(\omega_{2}+1\right)_{\delta}$ and $K=\beta\left(X \times \omega^{*}\right)$. As in $\S 2$, it suffices to show that $X \times \omega$ can be $C^{*}$-embedded in $\omega^{*}$. However since $X$ is Lindelöf and $\omega^{*}$ is an $F$-space it suffices to show that $X$ embeds in $\omega^{*}$. Since we are assuming $c>\omega_{1}$, we can embed $X$ in $2^{c}$. Now $X$ is a $P$-space, hence $X$ embeds in $\left(2^{c}\right)_{\delta}$ and by 3.2 in $\omega^{*}$.

We can now state our main results. 
3.4. THEOREM. The following are equivalent:

(i) $\mathrm{CH}$,

(ii) each open subset of a compact F-space of weight $c$ is an F-space,

(iii) each open subset of an F-space of weight $c$ is an F-space,

(iv) each locally compact subspace of an F-space of weight $c$ is an F-space.

Proof. Theorem 1.2, Theorem 1.3 and the example in $\$ 2$.

3.5. THEOREM $(\neg \mathrm{CH})$. Every infinite compact F-space contains a locally compact subspace which is not an F-space and has weight $c$.

Proof. Every infinite compact $F$-space contains $\omega^{*}$ and therefore, by Corollary 3.3, contains $K$.

\section{REFERENCES}

[vD1] E. K. van Douwen, $A$ basically disconnected normal space $\Phi$ with $|\beta \Phi \backslash \Phi|=1$, Canad. J. Math. 31 (1979), 911-914.

[vD2] __ P-spaces embed in $\beta \kappa$, Notices Amer. Math. Soc.

[vDvM1] E. K. van Douwen and J. van Mill, Parovičenko's characterization of $\beta \omega \backslash \omega$ implies $C H$, Proc. Amer. Math. Soc. 72 (1978), 539-541.

[vDvM2] _ Subspaces of basically disconnected spaces or quotients of countably complete Boolean algebras, Trans. Amer. Math. Soc. 259 (1980), 121-127.

[vDvM3] _ There can be $C^{*}$-embedded dense proper subspaces in $\beta \omega \backslash \omega$, (preprint).

[D] A. Dow, On F and $F^{\prime}$-spaces, Pacific J. Math (to appear).

[FG] N. J. Fine and L. Gillman, Extensions of continuous functions in $\beta N$, Bull. Amer. Math. Soc. 66 (1960), 376-381.

[GJ] L. Gillman and M. Jerison, Rings of continuous functions, Van Nostrand, Princeton, N.J., 1960.

[H] S. H. Hechler, On a ubiquitous cardinal, Proc. Amer. Math. Soc. 52 (1975), 348-352.

[L] A. Louveau, Caractererisation des sous-espaces compact de $\beta N$, Bull. Amer. Math. Soc. 97 (1973), 259-263.

[Pa] I. I. Parovičenko, A universal bicompact of weight $\boldsymbol{\aleph}$, Soviet Math. Dokl. 4 (1963), 592-595.

[Wa] R. C. Walker, The Stone-Čech compactification, Ergeb. Math. Grenzgeb., Bd 83, Springer-Verlag, Berlin, Heidelberg and New York, 1974.

[W1] R. G. Woods, The structure of small normal F-spaces, Topology Proc. 1 (1976), 173-179.

[W2] _ Characterizations of some $C^{*}$-embedded subspaces of $\beta N$, Pacific J. Math. 65 (1976), $573-579$.

[W3] A survey of absolutes of topological spaces, Topological Structures. II, Math. Centre

Tracts, No. 116, Mathematisch Centrum, Amsterdam, 1979, pp. 323-362.

Department of Mathematics, University of Manitoba, Winnipeg, Manitoba, Canada R3T 2N2

Current address: Department of Mathematics, University of Toronto, Toronto, Ontario, Canada M5S $1 \mathrm{~A} 1$ 